\title{
PASADO Y PRESENTE DE LAS REPOBLACIONES FORESTALES EN MONTES DE SOCIEDADES DE VECINOS
}

\author{
POR \\ SANTIAGO FERNÁNDEZ MUÑOZ Y RAFAEL MATA OLMO
}

Introducción: Las sociedades de vecinos en la cambiante política forestal

Este artículo se ocupa de algunos aspectos de las repoblaciones forestales del último medio siglo en los montes de sociedades de vecinos del Sistema Central, concretamente de la cuenca alta del río Sorbe, al norte de la provincia de Guadalajara. Dentro de un amplio proyecto de investigación sobre las consecuencias ecológicas y socioeconómicas de las denominadas repoblaciones históricas ${ }^{1}$, el objetivo de estas páginas es dar cuenta del papel desempeñado por las sociedades de vecinos en las operaciones repobladoras, comparando lo que fueron las actuaciones masivas de los años cincuenta a setenta, con la aplicación de las recientes medidas de reforestación de tierras agrícolas, de acompañamiento de la reforma de la P.A.C. aprobada en 1992.

${ }^{1}$ El proyecto lleva por título «Análisis de las repoblaciones históricas recientes e inventario de forestaciones actuales. Aspectos territoriales, ambientales, soicoeconómicos y culturales» (Programa sectorial I+D Agrario y Alimentario).

Santiago Fernández Muñoz y Rafael Mata Olmo: Departamento de Geografía. Universidad Autónoma de Madrid.

Estudios Geográficos

Tomo LXI, 2000, n. ${ }^{\circ} 240$, julio-septiembre 
Además de aportar información sobre una iniciativa concreta, el estudio empírico llevado a cabo es una buena ocasión para reflexionar, arrancando del caso que nos ocupa, sobre el cambiante significado de los montes de las sociedades de vecinos en una etapa de profunda transformación socioeconómica y política a todas las escalas, y sobre la forma en la que el tratamiento de los montes vecinales se incardina en los diferentes discursos reforestadores del periodo considerado. Se trata, en esa línea, de comparar el papel, los objetivos, los métodos y los resultados sociales y ecológicos de dos periodos, dos políticas y dos técnicas de intervención forestal, vinculando en cada caso los discursos y los procedimientos técnico-administrativos de carácter general con realidades locales en continua y profunda mutación.

Para ello se han seleccionado, como ejemplos y como laboratorio, dos montes limítrofes de los municipios de Arbancón y Cogolludo, situados en el Alto Sorbe (Sierra de Ayllón ${ }^{2}$, provincia de Guadalajara -figura 1), que permiten comparar dos espacios gestionados por sociedades de vecinos con resultados claramente diferentes: el actual monte de la Junta de Comunidades de Castilla La Mancha, Robledo y Otros/Jócar — Gu-1019_, repoblado en su totalidad en el período 19511974, y el Barranco de Valdemarojal (figura 2), que ha permanecido con aprovechamiento vecinal de pastos hasta 1992, año en que comenzó su repoblación por iniciativa de la entidad propietaria, acogiéndose a las medidas reforestadoras de la reforma de la P.A.C.

Las sociedades de vecinos, definidas jurídicamente como comunidades de bienes de origen vecinal (Martín Retortillo, 1961), son en la generalidad de los casos asociaciones de habitantes de un municipio, constituidas tras el proceso desamortizador con objeto de adquirir y conservar la propiedad y el uso colectivo de parte de los montes de propios enajenados por el Estado en la desamortización.

En otras zonas del Sistema Central ya estudiadas (Manuel Valdés, 1995; Sáez Pombo, 1998; Llorente Pinto, 1995) y en la Sierra de Ayllón, que ahora nos ocupa, los bienes de propios suponían un elevado porcentaje de la superficie total de montes, resultando fundamentales en la organización y funcionamiento de las comunidades rurales, pues de

${ }^{2}$ La totalidad de los documentos y publicaciones consultadas incluyen la zona en la Sierra de Ayllón por lo cual hemos optado también por utilizar este topónimo, aunque realmente sería más correcto denominar estas estribaciones del Sistema Central como Sierra del Ocejón. 


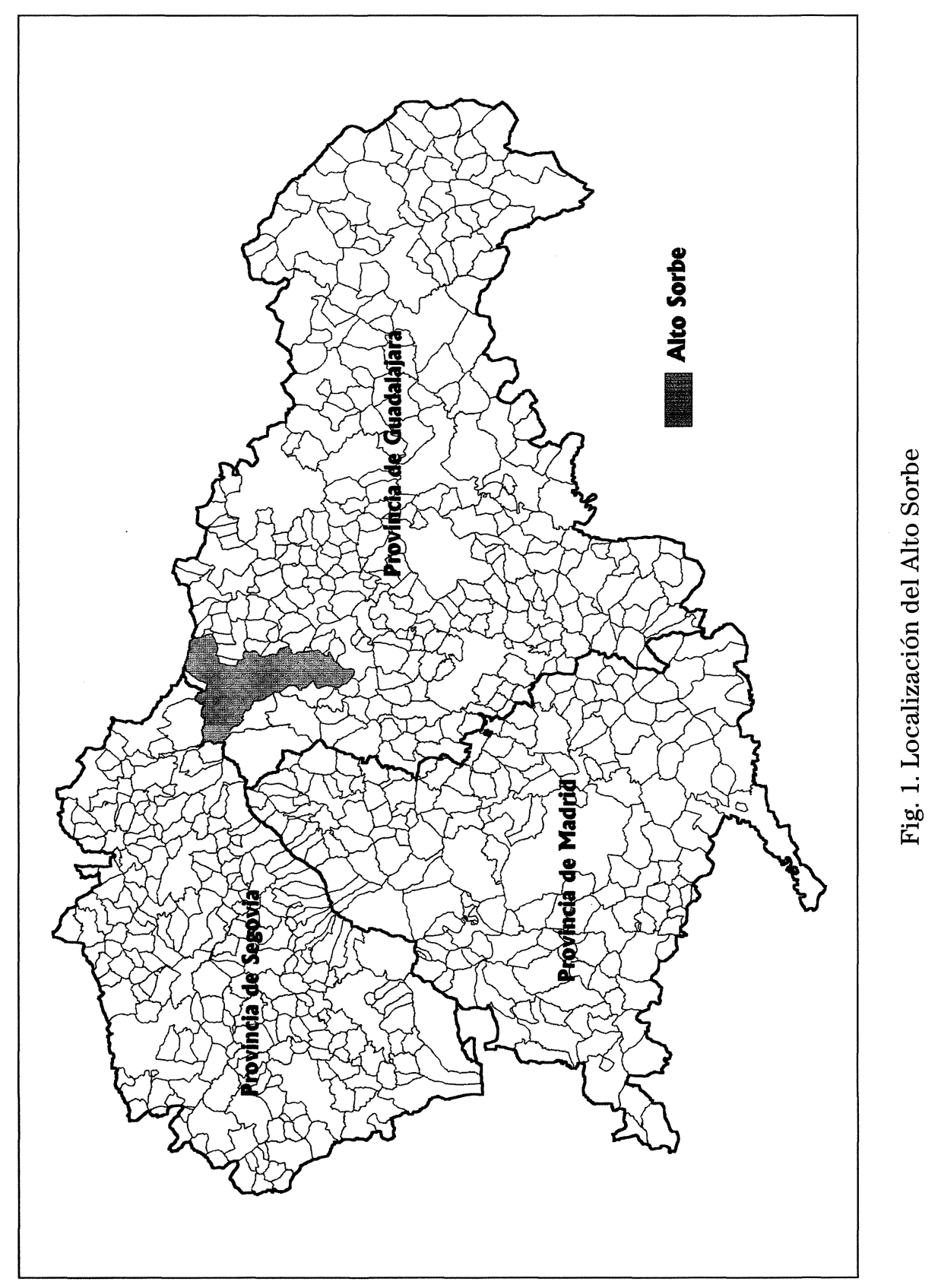

$-463-$ 


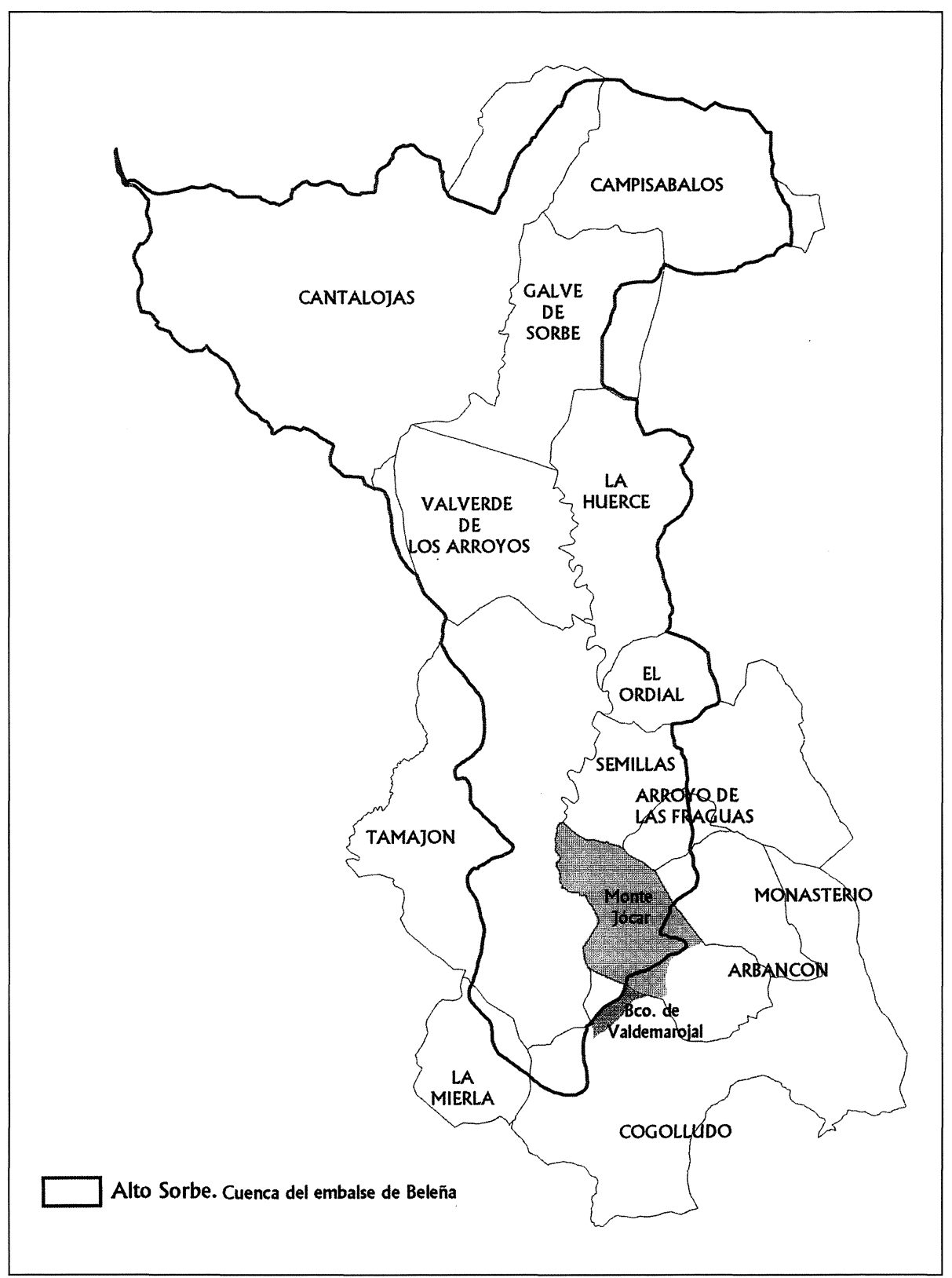

Fig. 2. Situación de los montes analizados en el Alto Sorbe.

$$
-464-
$$


ellos procedía una parte variable, pero en todo caso importante, del combustible, los recursos pastables, la madera en algunos casos, y otros aprovechamientos, como pequeñas cosechas de grano por el sistema de rozas, conocidas como centeneras en nuestro ámbito de estudio (López Gómez, 1974). Eran, pues, espacios vitales y de equilibrio para las economías locales.

Las rozas, el carboneo y el aprovechamiento ganadero ancestral habían reducido notablemente la cubierta forestal de estos montes, hasta su práctica desaparición en muchos casos. De ese modo, cuando sobrevino la desamortización civil y las normas complementarias de excepciones de ventas, fueron numerosos los montes concejiles que por carecer del arbolado que establecía la norma- «pino, roble o haya»- no pudieron ser exceptuados de venta (Fidalgo, 1987). Los usuarios tradicionales se vieron así privados del acceso a un recurso tradicional, enajenado y privatizado a favor de intereses muchas veces ajenos a la zona.

Pues bien, las sociedades de vecinos surgen, justamente, como una respuesta local y colectiva a la privatización de parte del patrimonio concejil tradicional. Por lo que sabemos, en el Alto Sorbe un elevado porcentaje de los espacios desamortizados terminaron figurando a nombre de vecinos de los pueblos - aunque no de todos los vecinos, como antaño-, en la mayor parte de las ocasiones bajo la fórmula de sociedades de vecinos.

El procedimiento habitual consistía en que los predios subastados fueran adquiridos por un intermediario que posteriormente los traspasaba a los vecinos; este es el caso de los montes Mojón Blanco y Canaleja (término municipal de Galve de Sorbe) rematados en favor de Eduardo Caballero en 1870 y traspasados a la Sociedad de Vecinos de Valverde de los Arroyos y Valdepinillos dos años después (Archivo Municipal de Valverde, A.M.V. 1924). En algunas ocasiones dicho intermediario era un vecino del pueblo que abona los correspondientes pagarés para obtener la propiedad definitiva de los montes y, posteriormente, reconoce que los verdaderos compradores son un elevado número de convecinos. Así ocurrió con los montes Ocejón, Umbría Gorda, Peyuste, Rincón y Dehesa del Sonsaz (municipio de Valverde de los Arroyos), comprados por Manuel Monasterio en subasta pública, aunque actuando como testaferro de más de un centenar de habitantes del pueblo, según se admite ante la autoridad judicial en 1903 (A.M.V. 1903). También se han documentado ca- 
sos en los que el primer comprador realizaba el capital forestal de la finca y posteriormente traspasaba el predio a la sociedad de vecinos. Así ocurrió con los montes de Abajo, Tras el Lomo, Cerrajo y Piedra Blanca de Tamajón, comprados por el senador Julián López Chavarri en segunda subasta en 1874, quien tras descuajar las «13.600 encinas seculares de cientotreinta centímetros de circunferencia» (Archivo Histórico de Guadalajara, A.H.G., 1874), los vendió en 1891 a una sociedad de vecinos formada por 112 habitantes de la villa.

Se ha podido constatar que en la práctica totalidad de los municipios de la comarca se constituyeron sociedades de vecinos tras la desamortización ${ }^{3}$, quedando demostrada su importancia en Tamajón, donde los bienes escriturados a favor de estas organizaciones supusieron al menos 2.447 ha (Archivo Municipal de Tamajón, A.M.T. 1891), en el antiguo municipio de Palancares, en el que totalizaron 1.523 ha (ICONA 1975) o en el de Valverde de los Arroyos y Valdepinillos donde alcanzan las 4.900 ha (A.M.V. 1903).

Los antiguos bienes de propios se escrituraron «en común y proindiviso» a favor de los miembros de la sociedad de vecinos; es decir, la propiedad correspondía al conjunto de los integrantes de la sociedad, sin que en ningún caso se delimitasen o adjudicasen partes o lotes concretos a cada uno de ellos; no obstante, en los casos de las sociedades de Tamajón (Sociedad de Baldios de Tamajón), Arbancón (Sociedad de Baldios de Valdemarojal) y Valverde de los Arroyos (Sociedad de Vecinos de Valverde), los vecinos no contaban con igual número de partes, lo cual otorgaba asimismo distintos derechos de aprovechamiento. También en la Dehesa de Cespedones, enajenada por la administración en 1887 (A.H.G., 1887) y adquirida por la Comunidad de Vecinos de Palancares (A.M.T. 1976), los vecinos propietarios disponían de diferentes extensiones en los baldíos (LiBRo..., 1939).

En definitiva, las sociedades de vecinos permitieron, como destaca Ester Sáez, que el «cambio de titularidad de los comunes [no] alterara substancialmente sus aprovechamientos» (Sáez, 1998, 487), pues continuaron siendo los propios vecinos, al menos una parte de ellos, los que poseían y gestionaban los antiguos bienes de propios.

\footnotetext{
${ }^{3}$ Los archivos municipales y los expedientes de expropiación de montes de la década de los setenta permiten confirmar que existían sociedades de vecinos en los antiguos municipios de Almiruete, Cantalojas, La Huerce, Galve de Sorbe, Jócar, Santotís, Tamajón, Valverde de los Arroyos y Umbralejo.
} 
Las primeras repoblaciones en al Alto Sorbe

La ubicación de la cuenca alta del río Sorbe, relativamente próxima a Madrid, determinó que sus aguas fueran reservadas ya desde 1921 (Real Orden de 12 de septiembre de 1921) para el abastecimiento de la capital, ante el «considerable aumento que sin interrupción vienen experimentando las cifras de la población de Madrid y del consumo de agua por habitante», prohibiéndose la tramitación de cualquier expediente de aprovechamiento de sus aguas. A partir de entonces la administración desarrolló diferentes proyectos de regulación de la cuenca, de forma que en 1940 se encontraba ya en estudio la construcción de un embalse en la zona de Beleña de Sorbe.

El conjunto de las repoblaciones previstas y desarrolladas en la zona tenían como principal objetivo corregir los fenómenos erosivos y proteger de esa manera la capacidad de los futuros embalses. Así se manifiesta en el primer documento redactado por la administración forestal (4. ${ }^{a}$ División Hidrológico Forestal) sobre el conjunto del Alto Sorbe en 1943 (Memoria de Reconocimiento General de la Cuenca del río Sorbe ${ }^{4}$ ), en el que se propone la repoblación general de la cuenca, «con el fin de regularizar el régimen de las aguas y evitar las turbias», pues pese a reconocerse que la erosión no es muy acusada, sí lo es potencialmente, «ya que las fuertes pendientes de un terreno tan sumamente montañoso y erosionable, sometido a un clima rudo, convertirían fácil y rápidamente esta cuenca en una zona llena de deslizamientos y con una inmensa red de torrenteras que producirían daños de consideración» (P.F.E., 1943).

No debe desdeñarse tampoco entre las razones que justificaron las repoblaciones en el ámbito de estudio, como en otras muchas zonas de España, la consideración de que los espacios de montaña forman parte de una región forestal cuyo uso vocacional es el bosque; así, en la $\mathrm{Me}$ moria se afirma que toda la cuenca esta incluida «dentro de la zona típicamente forestal» y que, por tanto, la mayor parte de su superficie es «terreno impropio para el cultivo agrícola» (P.F.E., 1943). La necesidad de regenerar unos montes, «profundamente degradados» debido a los «aprovechamientos abusivos de maderas, leñas, roturaciones y pasto-

${ }^{4}$ Redactada de acuerdo con lo establecido en las Instrucciones de Repoblación Forestal aprobadas por Decreto del Ministerio de Agricultura de 12 de julio de 1933. 
reo» es, pues, otro de los argumentos que conduce a la propuesta de repoblar una parte importante de la cuenca.

Para poner en práctica las repoblaciones era preciso que la administración forestal, en este caso el Patrimonio Forestal del Estado (P.F.E.), se hiciera con el control de los predios a reforestar, para lo cual escogió la vía del Consorcio. Como es sabido, se trataba de contratos firmados entre el Patrimonio Forestal del Estado y la entidad propietaria del monte según los cuales la propiedad del suelo permanecía en manos de sus titulares, en tanto que el vuelo era transferido al Patrimonio hasta que la masa creada alcanzase el turno de corta. Entre 1947 y 1957 se consorciaron en el Alto Sorbe ocho montes ${ }^{5}$ (figura 3), sobre una extensión total de 7.700 ha. La mayor parte de los consorcios se establecieron en la zona septentrional de la cuenca (municipios de Cantalojas y Valverde de los Arroyos), pese a reconocerse que la erosión era poco significativa. Asimismo se suscribieron dos en la zona meridional (montes de Jócar y Semillas) con el objetivo principal de controlar la erosión laminar y de fondo.

Uno de los primeros consorcios en constituirse fue el que afectó a 1.000 ha «de terrenos comunales» del monte Robledo y otros, de Jócar, pobladas, según los documentos de la administración forestal, de jaras y estepas (Cistus ladaniferus y laurifolius), así como por algún ejemplar de enebro (Juniperus oxicedrus), aliagas (Genista scorpius) y espliego (Lavandula stoechas). Con el fin de contrastar esta información y delimitar de forma precisa los usos del suelo del conjunto del antiguo municipio de Jócar, agregado en 1940 a Arbancón, se ha realizado la fotointerpretación ${ }^{6}$ del recientemente rescatado vuelo Americano de la Serie A, llevado a cabo en 1946.

La utilización de un Sistema de Información Geográfica ha permitido georeferenciar las fotografías aéreas y obtener el mapa de usos en 1946 (figura 4) en el que efectivamente se constata la intensa deforestación de la zona, ocupada en su mayor parte por matorral para aprovechamiento caprino, y con presencia significativa de terrenos rozados y cultivados (las centeneras). Corresponde esta distribución de aprovechamientos a un mo-

\footnotetext{
${ }^{5}$ Los montes consorciados fueron Robledal de la Sierra, en el que se establecieron tres consorcios, Tejera Negra y Renovizal, Ocejón, Cuartel de la Mellizas, Semillas y Robledo y otros (Jócar).

${ }_{6}^{6}$ Realizada por Nieves López Estebanez y Santiago Fernández Muñoz.
} 


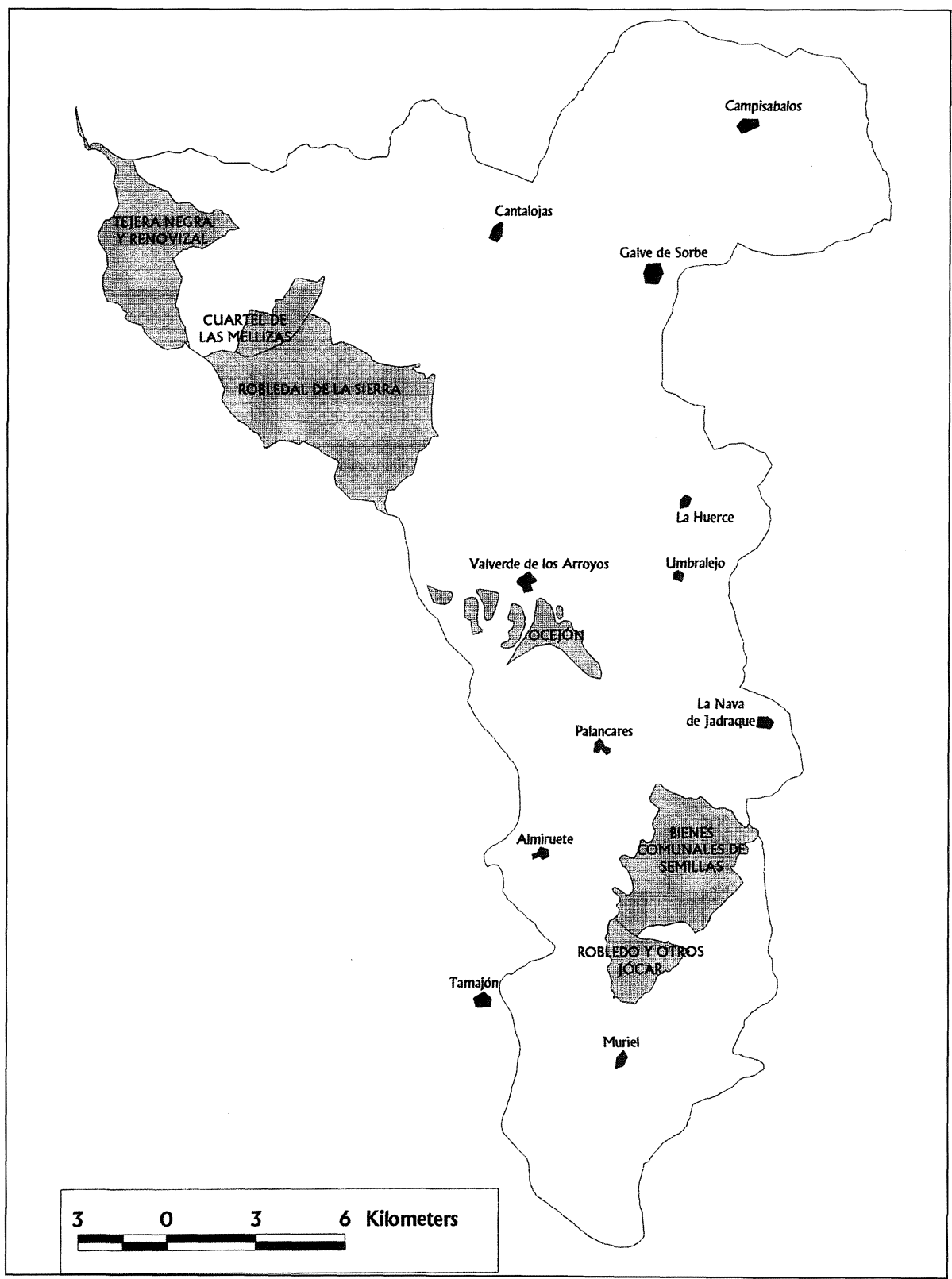

Fig. 3. Montes consorciados en el Alto Sorbe. 1947-1957.

$-469-$ 


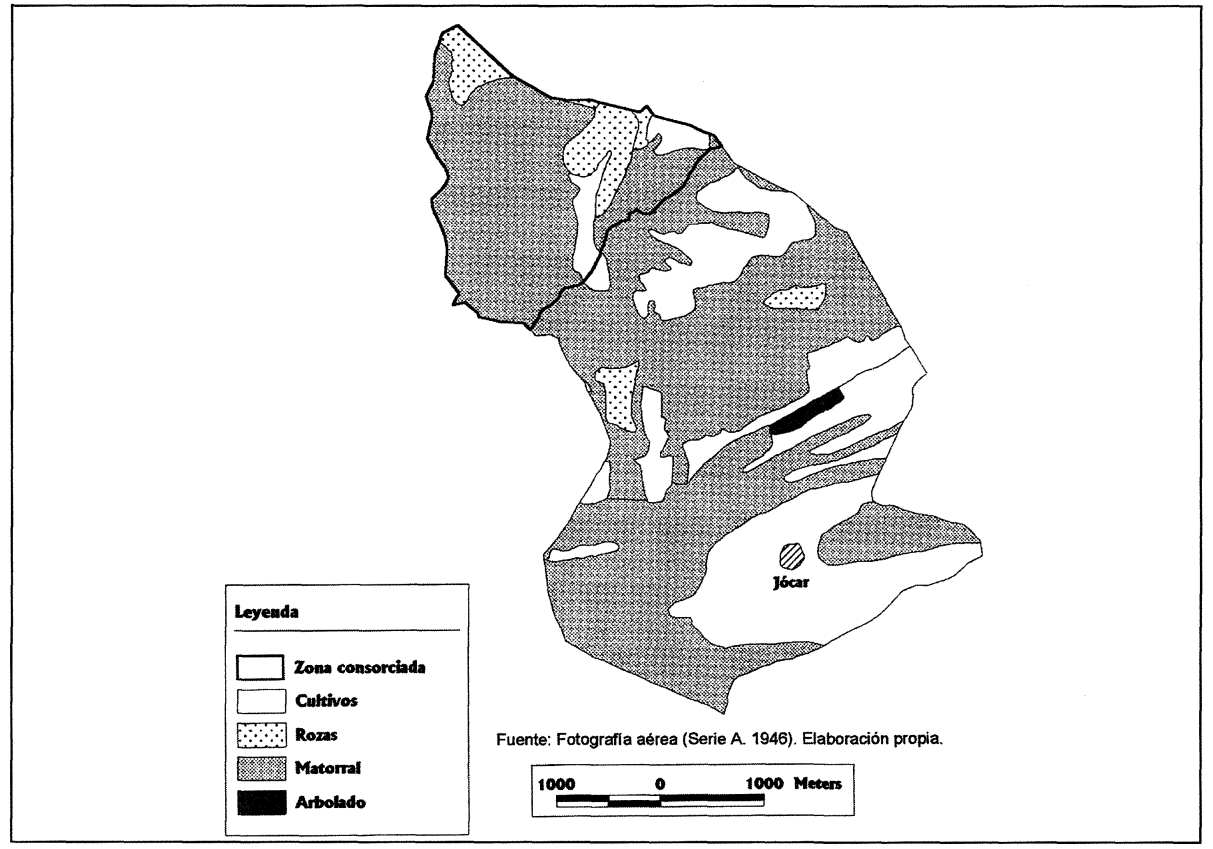

Fig. 4. Monte Jócar. Usos del suelo en 1946.

delo de gestión de amplias zonas de la montaña media peninsular en momentos de máxima carga demográfica, en los que a la vocación pastoril tradicional se une la roturación para la obtención de cosechas temporales de grano. Al arbolado le quedaba en esta organización de los usos y aprovechamientos un puesto muy secundario o inexistente.

USOS DEL SUELO DE LA ZONA CONSORCIADA 1946

\begin{tabular}{lr}
\hline \multicolumn{1}{c}{ Usos del suelo } & Porcentaje \\
\hline Matorral & 75,3 \\
Rozas & 16,4 \\
Cultivos & 8,3 \\
Total & 100
\end{tabular}

Fuente: Elaboración propia a partir de fotointerpretación. 
El consorcio se realizó en 1951, cuando aún no se había iniciado la masiva emigración de los pueblos de la Sierra de Ayllón, que tiene mayoritariamente lugar a fines de los cincuenta y en la década siguiente; así, la población del conjunto del municipio de Arbancón (incluido Jócar) sólo había descendido en 14 habitantes entre 1940 y 1950 (pasó de 562 habitantes a 548), siempre según datos oficiales.

El papel de la sociedad de vecinos en el establecimiento del consorcio no ha podido ser aclarado por completo, pues no se conserva documentación administrativa al respecto. No obstante, a partir de lo ocurrido en el Alto Sorbe y tras las entrevistas realizadas a habitantes de los pueblos vecinos cabe hacer algunas consideraciones. En primer lugar se puede comprobar que la mayor parte de los consorcios establecidos por la administración forestal en la zona eran suscritos con los Ayuntamientos ${ }^{7}$, lo que no siempre responde a que los montes perteneciesen a los municipios; de hecho Sáez $(1998,479)$ ha podido comprobar en los cercanos montes de La Puebla (Madrid), similares ecológica y socialmente a los que aquí nos ocupan, cómo se materializa la municipalización de los espacios de las sociedades de vecinos al ser el consistorio el que establece los consorcios, pese a no ser el propietario de los terrenos. Un proceso similar se produjo con el monte Ocejón de Valverde de los Arroyos cuyo consorcio fue firmado por el Ayuntamiento pese a pertenecer a la Sociedad de Vecinos de la localidad o con el predio Tras el Lomo y otro, consorciado por el Ayuntamiento de Tamajón en 1951, hecho impugnado por la Sociedad de Baldios en 1964, argumentando que legalmente el consistorio no disponía de ningún derecho sobre el monte.

Otro aspecto que conviene destacar es que los consorcios afectan únicamente a una parte de los terrenos de aprovechamiento común; concretamente en el caso de Jócar se establece el consorcio sobre las áreas más alejadas del núcleo de población, de forma que los vecinos pudieron mantener el aprovechamiento ganadero y las rozas al menos sobre un sector de sus antiguos bienes de propios. Sin embargo, no se puede descartar que el consorcio fuera rechazado por parte de los vecinos, tal y como se ha podido comprobar en otros establecidos en los municipios de Cantalojas, Semillas y Tamajón.

${ }^{7}$ En el caso del monte Robledo y otros el Patrimonio establece el consorcio con el Ayuntamiento de Arbancón. 
En el caso de Cantalojas, poco tiempo después de aprobados dos consorcios en el actual monte Los Cuarteles (Gu-1027), fue preciso delimitar un perímetro de repoblación obligatoria debido a «la negativa de los propietarios de los cuarteles a repoblar» (P.F.E., 1955), y pese a que en el decreto de aprobación del perímetro se establecía la declaración de utilidad pública y necesidad y urgencia de la repoblación forestal, su «ejecución se vio interrumpida por causas de tipo social, ya que los acotamientos al pastoreo, necesarios para hacer la repoblación, han hecho imposible el continuar los trabajos iniciados» (P.F.E., 1968). Por su parte, el ayuntamiento de Ayllón en representación de la Mancomunidad de Ayllón, entidad propietaria del monte consorciado Tejera Negra y Renovizal, solicita en 1955 «compensación de renta perdida» a la administración como consecuencia de las vedas al pastoreo impuestas en el monte (P.F.E. 1951a).

La oposición de los vecinos a las repoblaciones se constata también en Semillas, cuya ayuntamiento había suscrito en 1952 un consorcio sobre 2.000 ha de los montes comunales y que, sólo dos años después y cuando sólo se habían repoblado 50 ha, remite una instancia al Distrito Forestal manifestando su disconformidad con la situación creada. El escrito de la corporación municipal, apoyado por una misiva de los ganaderos de la localidad, advierte que «se consorciaron una cantidad de hectáreas que no existen para la repoblación» por lo cual se solicita su reducción a las «500 ha disponibles para tal fin», así como que no se repueble un segundo paraje mientras el ganado no paste en el predio repoblado «ya que de lo contrario se perjudicará grandemente a la ganadería que es el único medio de ingresos que los vecinos disponen» (P.F.E. 1952). También la Sociedad de Baldíos de Tamajón, pese a que acepta establecer un consorcio con el Patrimonio Forestal del Estado sobre un monte de su propiedad, solicita le sea permitido introducir ganado en parte del mismo debido a que consideran «la zona suficientemente repoblada de arbolado, en un término de tres mil hectáreas, de las cuales queda un número insuficiente para sostener la poca ganadería que existe en la actualidad, base fundamental para el sostenimiento de habitantes del pueblo» (A.M.T. 1964).

En lo que se refiere a Jócar, no se ha encontrado ningún documento que permita establecer la posición de los vecinos respecto al consorcio, y si bien las referencias de vecinos de pueblos cercanos no recuerdan que hubiera conflictos, tampoco es descartable que, como en los casos 
de las localidades vecinas, los propietarios de ganado presentasen resistencia a perder pastizales. En todo caso, las repoblaciones comenzaron en 1952 en la zona limítrofe con el municipio de Semillas, con el descuaje del matorral y la plantación por ahoyado manual de Pinus pinaster realizada por una cuadrilla de obreros traídos desde Ávila. La superficie repoblada fue pequeña en los primeros años, hasta que en 1959 se abandonó el método de ahoyado y se comenzó a plantar Pinus pinaster y nigra sobre banquetas o terrazas de una anchura mínima de 1 $\mathrm{m}$, construidas según curvas de nivel por yuntas de bueyes. A partir de 1965 las terrazas comenzaron ya a ser trazadas por maquinaria forestal, incrementándose notablemente su amplitud.

Poco después de comenzar las primeras repoblaciones, el Gobierno aprobó un decreto por el cual quedaron «reservados para su único empleo en el abastecimiento de agua potable a Madrid y pueblos próximos, los caudales del Sorbe hasta el Estrecho de Pozo de los Ramos», mientras que las aguas sobrantes serían reguladas por un nuevo embalse que serviría para «la ampliación de los abastecimientos de aguas potables de Alcalá de Henares y Torrejón (...) y a los de Guadalajara y pueblos próximos» (Decreto de 10 de agosto de 1954. BOE 14 septiembre). Los proyectos de las dos presas a construir en el Sorbe se retrasaron hasta 1961, año en el que se aprobó el «Anteproyecto de embalses de regulación en el río Sorbe», que proponía construir dos represamientos, uno en la cerrada de Pozo de los Ramos y otro en las cercanías del pueblo de Beleña de Sorbe. El proyecto de construir este último embalse fue el origen de la decisión de la administración forestal de repoblar todos los terrenos cuyas aguas vertieran directamente a él, con el fin de reducir la llegada de arrastres sólidos al vaso del embalse.

Una pequeña parte de los baldíos del antiguo término municipal de Jócar eran drenados por arroyos que desembocaban en el futuro embalse, por lo que se declaró un perímetro, que no sólo establecía la repoblación obligatoria y expropiación forzosa las zonas situadas en la cuenca alimentadora del embalse de Beleña, sino que extiende esta consideración al conjunto de los terrenos de la entidad menor, afectando a una superficie de 2.152 ha, que incluían las 822 ha ya repobladas en la zona consorciada y reservaba para uso agrícola $343 \mathrm{ha}$.

El perímetro de repoblación fue aprobado por el decreto 3272/67 de 21 de Diciembre (BOE, 15 enero de 1968) y en poco tiempo se procede a la expropiación forzosa de todos los terrenos de Jócar, incluida la men- 
cionada superficie de uso agrícola. En este caso no sólo se adquieren los terrenos propiedad de la sociedad de baldíos del pueblo, sino también la totalidad de las propiedades diferenciadas de la entidad menor.

La pregunta que se suscita, a tenor del objetivo de este artículo, es cuál fue el papel de los miembros de la sociedad de vecinos, titulares en común y proindiviso de una buena parte de los terrenos a expropiar. En primer lugar hay que destacar que entre la firma del consorcio en 1951 y la declaración del perímetro de repoblación obligatoria se había producido un notable descenso de la población del término municipal de Arbancón (no es posible contar con datos concretos de Jócar); los habitantes censados pasan de los mencionados 548 de 1950 a 488 en 1960 y a 238 en 1970, siendo la reducción relativa de un $62 \%$ desde 1930 .

Otro elemento que debe considerarse es que, según la memoria justificativa del decreto de repoblación forestal obligatoria (P.F.E. 1967), fueron los propietarios los que iniciaron «gestiones para la venta de los mismos al Patrimonio Forestal del Estado». Las entrevistas a vecinos de la zona confirman este extremo, al tiempo que permiten afirmar que fueron antiguos habitantes del pueblo establecidos ya en ámbitos urbanos, con una cierta formación y patrimonio rústico algo más crecido, los que llevaron las negociaciones con la administración forestal. Hay aquí, por tanto, un asunto de estratificación social, por mínima que fuera, en el que convendrá profundizar para aportar alguna luz sobre las relaciones que se establecen entre los poderes central y local en relación con la repoblación forestal, en un contexto férreamente autocrático y altamente jerarquizado. La memoria señala también que «el poblado [Jócar] se encuentra despoblado» en 1967, afirmación desmentida en las entrevistas; así, parece que si bien un elevado porcentaje de la población emigró con anterioridad a la aprobación del Decreto, quedaba en el pueblo un reducido número de vecinos que pretendían permanecer y mantener sus actividades, no pudiéndose precisar si fue la presión de la mayoría de los antiguos habitantes, de la administración forestal, o de ambas a un tiempo - como parece probable-, lo que indujo la enajenación a favor del Patrimonio de todos los terrenos.

Ultimada la adquisición de todas las fincas comenzó su repoblación con Pinus pinaster y nigra de una savia, sobre terrazas construidas según curvas de nivel por tractores de cadenas. El resultado final fue la forestación de la práctica totalidad del antiguo municipio de Jócar y la suplantación de una entidad serrana eminentemente agropecuaria, con 
peso importante de formas de organización colectiva, por un espacio homogéneamente arbolado, poseído y gestionado por el Estado. La «destacada» participación de las sociedades de vecinos en esta fase de «cruzada» repobladora no supuso otra cosa que su total desaparición. He aquí un excelente ejemplo de las idas y vueltas, y de algunas de las aparentes contradicciones de nuestra reciente historia forestal, que en cualquier caso sólo pueden ser interpretadas en sus particulares contextos temporales (políticos y socioeconómicos) y espaciales: propiedad y aprovechamiento concejil en origen, privatización de propios después, recuperación de la tenencia y usos colectivos por la sociedades de vecinos, y finalmente erradicación y estatalización de espacios antaño comunales.

REPOBLACIONES DEL MONTE JÓCAR

\begin{tabular}{|c|c|c|c|c|c|c|}
\hline \multirow{2}{*}{ Año } & \multicolumn{2}{|c|}{ Repoblación } & \multicolumn{2}{|c|}{ Marras } & \multicolumn{2}{|c|}{ 2. ${ }^{a}$ repoblación } \\
\hline & Especie & Superficie & Especie & Superficie & Especie & Superficie \\
\hline 1959 & P. nigra & 25 & & & & \\
\hline 1961 & P. nigra & 105 & & & & \\
\hline 1962 & P. nigra, pinaster & 50 & & & & \\
\hline 1963 & P. nigra, pinaster & 80 & & & & \\
\hline 1963 & P. pinaster & 46 & P. nigra & 45 & & \\
\hline 1964 & P. pinaster & 16 & P. pinaster & 41 & & \\
\hline 1966 & P. pinaster & 50 & & & & \\
\hline 1967 & P. pinaster & 150 & & & P. pinaster & 50 \\
\hline 1969 & P. pinaster & 300 & P. pinaster & 110 & & \\
\hline 1970 & P. nigra, pinaster & 600 & & & & \\
\hline 1971 & P. nigra, pinaster & 452 & P. pinaster & 70 & & \\
\hline 1972 & & & P. pinaster & 93 & & \\
\hline 1973 & & & P. pinaster & 170 & & \\
\hline 1975 & & & & & P. pinaster & 24 \\
\hline Total & & 1.874 & & 529 & & 74 \\
\hline
\end{tabular}

Fuente: Inventario de repoblaciones. 1992. Distrito Forestal de Guadalajara

Nuevas iniciativas de reforestación y respuesta de las sociedades de vecinos: estudio de un caso

Las divisiones administrativas o aquellas que se establecen en cualquier tipo de planificación tienen en muchos casos consecuencias espaciales relevantes. En este sentido, en el ámbito del Alto Sorbe, la 
pertenencia a cuencas hidrográficas diferentes o a distintos tramos de las mismas, ha determinado que terrenos de similares características biofísicas y productivas hayan tenido usos y dinámicas muy diferentes. Así ocurre al comparar la evolución del paisaje de los montes limítrofes del Barranco de Valdemarojal y el de Jócar, ya tratado.

El Barranco de Valdemarojal, un monte de 140 ha situado en el antiguo municipio de Aleas (actualmente agregado a Cogolludo), fue adquirido durante el proceso desamortizador por Francisco Jareño, quien a su vez lo vendió, en común y proindiviso, a noventa y nueve vecinos del vecino pueblo de Arbancón, constituidos en la Sociedad de Baldíos de Valdemarojal. Esta sociedad adquirió también la finca denominada Barranco de Valdelafuente, así como un pequeño monte de 43 ha en el municipio de Semillas, siendo actualmente propietaria de 440 ha de monte en las áreas cercanas al municipio de Arbancón.

De forma análoga a lo referido sobre la adquisición de los baldíos de Tamajón y Valverde de los Arroyos, no todos los vecinos disponen de igual número de partes o acciones en la propiedad. De hecho se ha podido comprobar que, de los 63 miembros que aparecen actualmente como propietarios de la totalidad de los Baldíos, 43 poseen una o dos partes (cada parte equivale a $1,55 \mathrm{ha}$ ), frente a un vecino que dispone de más de 6 acciones, con 15,5 ha.

Por lo que se refiere a las características del monte, su base geológica es similar al área meridional de Jócar y presenta también pendientes pronunciadas (en un $80 \%$ de su superficie son superiores al 30\%). Asimismo, según se ha podido comprobar tras la fotointerpretación del vuelo de 1946, los usos del suelo eran muy parecidos a los existentes en Jócar, pues la mayor parte del suelo aparece ocupado por matorral, con algunas zonas rozadas.

Los vecinos entrevistados confirman lo observado en las fotografías aéreas y hablan de un jaral, aprovechado por el ganado cabrío de los miembros de la Sociedad de Baldíos. No se conservan documentos que permitan cifrar exactamente la carga ganadera del monte, sin embargo, las referencias orales coinciden en que, desde mediados de la década de los cincuenta, aprovechaba los pastos del Barranco de Valdemarojal un número cercano a las 300 cabras, conducidas al monte por un único pastor contratado por la Sociedad, carga que no se habría visto afectada por el descenso demográfico del lugar. Asimismo se ha podido constatar que anualmente se extraía un volumen 
indeterminado de leñas y se rozaban pequeñas parcelas para obtener cosechas de centeno.

Todos los elementos expuestos conducen a la conclusión de que no existen diferencias significativas en cuanto a propiedad, medio físico y aprovechamientos que justifiquen que el término de Jócar fuera repoblado y expropiado en el período 1951-1974, mientras que el Barranco de Valdemarojal mantiene la cubierta y los usos tradicionales hasta la actualidad. El único elemento diferenciador, pero fundamental desde el punto de vista de la política repobladora, es que las aguas de los arroyos de Jócar vierten al río Sorbe aguas arriba del emplazamiento de la presa de Beleña, mientras que el arroyo de Valdemarojal se une al Sorbe inmediatamente aguas debajo de la presa (figura 2), lo que provocó que la zona de Jócar fuera incluida entre las áreas a repoblar para proteger la capacidad del vaso del embalse, mientras que el Barranco de Valdemarojal permanecía ajeno al interés de la administración forestal.

La importancia de los aprovechamientos comunales del monte de Aleas fue descendiendo paulatinamente, abandonándose en primer lugar la práctica de las rozas y posteriormente la saca de leñas, hasta que finalmente, en una fecha no precisada pero que se puede fijar entre mediados de la década de los setenta y los primeros ochenta, se abandonó también la actividad ganadera.

El monte permanecía prácticamente sin aprovechamientos hasta que, en 1993, tiene lugar un hecho que va a modificar sustancialmente la gestión del predio y que es ilustrativo de las nuevas formas en que patrimonios de naturaleza y uso tradicionalmente colectivos se incorporan a los recientes discursos y políticas agrarias, forestales y, en última instancia, territoriales. Aquel año se convoca una reunión de los miembros de la Sociedad de Baldíos de Valdemarojal con el fin de acogerse a las subvenciones comunitarias establecidas en el Reglamento 2080/92 para fomentar la forestación en las explotaciones agrarias. Se pone de manifiesto aquí la pérdida de importancia productiva de los espacios forestales, pues mientras en los años cincuenta los consorcios realizados en la zona cuentan siempre con la oposición explícita de los ganaderos (Cantalojas, Tamajón, Semillas), en la década de los noventa son los propios vecinos quienes solicitan su repoblación con el fin de obtener algún beneficio del monte.

La citada medida comunitaria, como es bien sabido, tenía y tiene el carácter de acompañamiento de la reforma de la P.A.C., con el prioritario 
objetivo de proporcionar alternativas de uso y renta a tierras agrícolas mayoritariamente generadoras de excedentes. No es, al menos en origen, una medida forestal sino claramente agrícola. Se produce así, en un contexto político y socioeconómico muy distinto del de los años 50-70, un encuentro a escala local entre una forma «relicta» de tenencia y de paisaje, carente de cualquier función productiva actual, con una política «global», concebida y prevista para agriculturas excedentarias. La aplicación de estas políticas globales a las realidades administrativas y espaciales de los muy diversos territorios comunitarios permite que una sociedad de vecinos, superviviente de una compleja historia forestal, utilice o aproveche - si se prefiere esta expresión- unos fondos de reforestación que, en este caso concreto, van justamente dirigidos a terrenos con vocación claramente forestal y no a tierras propiamente agrícolas o pecuarias, como las previstas en el espíritu y la letra de la norma originaria.

La sociedad vecinal mantenía su existencia, pero sin actividad productiva, y con un perfil sociolaboral de sus miembros muy distinto al de veinte o treinta años atrás. De los 67 participantes en la decisión de acogerse a la reforestación de la P.A.C., un elevado número tiene su residencia en Madrid, Guadalajara y en otros municipios de la corona metropolitana madrileña; aunque son mayoría aún los que cuentan con domicilio en el pueblo - incluidos los jubilados-, ninguno hace uso de los recursos que ofrece la finca de la sociedad. El patrimonio inmueble se mantiene - con los gastos fiscales consiguientes-, el suelo conserva su cubierta dominante y tradicional de jara, pero no existe gestión alguna del monte.

Una interpretación muy abierta de la medida comunitaria permitió que la sociedad de vecinos se adhiriera a la iniciativa reforestadora en su primer año de aplicación (1994). La sociedad, previo acuerdo de sus miembros, solicitó a la Junta de Comunidades de Castilla-La Mancha la correspondiente subvención según lo establecido por la Orden de 13 mayo de 1993 (BOCM 19 de mayo), que trasladaba el reglamento español (Real Decreto 378/93) a la comunidad autónoma. Como se ha dicho, la disposición comunitaria tenía como objetivo fundamental retirar de la producción zonas agrarias y contribuir así a reducir excedentes. No obstante, el reglamento castellano-manchego establece también entre los fines de las subvenciones «corregir los graves problemas de erosión y desertización que sufren algunas comarcas y contribuir a una gestión del 
espacio natural más compatible con el equilibrio del medio ambiente, favoreciendo el desarrollo de ecosistemas forestales», ampliándose por tanto algunos de los argumentos que justificaron las repoblaciones en épocas anteriores. Por otra parte, el reglamento opta por una definición muy laxa de las superficies agrarias susceptibles de acogerse a las subvenciones, incluyendo prados naturales, pastizales y montes abiertos y dehesas, siempre que las copas del arbolado no cubran más del $20 \%$ del suelo y se utilice principalmente para pastoreo.

Como puede verse, la norma autonómica permite extender el concepto de explotación agraria a zonas de monte con clara vocación forestal, como el Barranco de Valdemarojal. La ayuda para la repoblación de las 140 ha de la finca fue concedida en 1994, suscribiéndose un contrato entre la Sociedad de Baldíos y una empresa sevillana por el que ésta se compromete a realizar la repoblación en dos años y a efectuar trabajos de mantenimiento y reposición de marras durante cinco, por un importe igual al concedido por la administración para la forestación. Las especies elegidas han sido Pinus pinaster en un $80 \%$ y Quercus ilex y Quercus faginea en el restante $20 \%$. Es de destacar que sólo el coste de la reforestación y de su mantenimiento en los cinco primeros años (alrededor de 27 millones de pesetas) supera con creces el valor del suelo de la finca.

Sin entrar en detalles y en el balance técnico de la operación, cosa que queda fuera de los objetivos de este texto, es de señalar que se han abandonado los aterrazamientos, procedimiento seguido en las repoblaciones efectuadas entre 1959 y 1974 en Jócar, de relevantes efectos paisajísticos y ambientales pues suponía la eliminación total de la vegetación y la creación de banquetas de importantes dimensiones. De hecho, se ha retomado el método de ahoyado empleado en las primeras forestaciones realizadas en la zona, aunque con obvias diferencias en cuanto a energía utilizada ya que, mientras en las plantaciones del período 1951-1956 el ahoyado y el desbroce era manual, hoy día estas operaciones están mecanizadas (Tractor de Alta Estabilidad). Las labores actuales de preparación del terreno únicamente eliminan el matorral en las fajas de plantación, realizándose posteriormente el subsolado, y el ahoyado mecánicamente. En las zonas de pendientes superiores al $50 \%$ no se realiza subsolado. No obstante, la idea de reforestar, de intervenir en el monte mediante la plantación de árboles es común a los proyectos desarrollados en la década de los sesenta y en los últimos años. 
Además de los gastos de repoblación, la ayuda comunitaria incluye una compensación por pérdida de renta para los propietarios de las zonas repobladas. Al estar integrada la Sociedad de Baldíos por un número elevado de propietarios, el ingreso que percibe cada uno de ellos es pequeño; no hay que olvidar, sin embargo, que hasta entonces los socios no percibían ingreso alguno y que la medida les está «compensando» por una merma de renta que en la realidad no se produce. La opinión generalizada de los socios es que el objetivo de la reforestación es «extraer algún beneficio al monte» 0 «al menos no perder dinero con él».

\section{Algunas ideas finales sobre el nuevo contexto y el nuevo sentido de las reforestaciones}

El contexto global y local de la intervención forestal en estos montes se ha modificado, pues, sustancialmente en los últimos 25 años. El único elemento de continuidad es el patrimonial, es decir, la pervivencia de grandes fincas de sociedades de vecinos - en nuestro caso concreto, el Barranco de Valdemarojal_, expresión casi arqueológica de formas de tenencia y uso colectivo tradicional. Ha desaparecido, sin embargo, el aprovechamiento que daba sentido a estos montes y, por tanto, su gestión; los miembros de la Sociedad son ya sólo propietarios, titulares de patrimonio, pero no usuarios.

Ese panorama podría justificar una intervención patrimonial «dura» por parte de las administraciones públicas (expropiación, compra), como las habituales hace algunos decenios en la zona, incluso sobre montes que eran objeto todavía de aprovechamiento. Pero el marco político es distinto en muchos sentidos: el escenario constitucional y democrático plantea las relaciones con los entes y los intereses locales de forma diferente a la del pasado autocrático, todo ello filtrado, interpretado y aplicado por las administraciones autonómicas. La creciente conciencia ambiental y, concretamente, la genérica y poco matizada crítica a las repoblaciones de tiempos pasados, dificulta el desarrollo de operaciones forestales del alcance y sentido de hace algunos decenios.

Por otra parte, la progresiva despoblación de las áreas de montaña y el abandono de los aprovechamientos tradicionales ha provocado que muchos espacios como el que nos ocupa hayan quedado marginados de la mayor parte de las medidas activas de política agropecuaria, y sin em-

$$
-480-
$$


bargo expuestos a la introducción de usos de elevado impacto, desarticuladores del paisaje y destructores, en muchos casos, de la riqueza biológica y cultural que encierran. En este sentido, la aplicación de la P.A.C. reforestadora en montes como el que nos ocupa es un eficaz instrumento para «exceptuar» espacios valiosos desde la perspectiva ecológica y/o paisajística de otros aprovechamientos potenciales conocidos ya en la zona, como la urbanización, la parcelación o los deportes de elevado impacto ambiental. La subvención por pérdida de renta puede ser contemplada entonces como una compensación a la sociedad de vecinos, que a costa de una lucha histórica por el suelo de aprovechamiento colectivo, conserva en la actualidad piezas significativas de monte.

Sin embargo, la intervención analizada, como otras muchas desarrolladas en España, al amparo de la normativa europea, en áreas marginales desde el punto de vista agrario y de vocación o dedicación forestal, debe ser valorada también a la luz de criterios ambientales y territoriales. Cabe cuestionar, en primer término, la oportunidad de la repoblación arbórea de montes en los que, como resultado del abandono de los usos tradicionales, se está produciendo un proceso de regeneración natural de la vegetación, con la existencia ya de un matorral de interés en términos de diversidad y de control de la erosión, dos circunstancias que junto con la compensación de pérdida de rentas agrarias figuran en la justificación del Reglamento comunitario. En áreas como estas no habría pérdida o descenso de rentas que compensar, con lo que los fines estrictamente ambientales (control de la erosión y fomento de la diversidad biológica) aparecen como el único apoyo de las actuaciones.

No se trata de negar la oportunidad de emprender cualquier tipo de intervención forestal de carácter arbóreo, pero sí de la necesidad de plantearlas con extraordinaria cautela en el caso de estimarlas necesarias. Es mucho lo que se ha aprendido a partir del análisis crítico de las denominadas «repoblaciones históricas»; se ha ampliado el conocimiento de la riqueza y diversidad florística de determinados matorrales y de su interés como habitats, ha crecido su valoración como paisajes expresivos de una determinada realidad geoecológica, se han modificado también los criterios de manejo de la cubierta vegetal en relación con las dinámicas de vertientes y se han podido evaluar los resultados de las plantaciones. Es cierto que el Reglamento 2080/92 enfatiza en la idea de 
«fomentar inversiones forestales en explotaciones agrarias» mediante la plantación de árboles; pero la norma comunitaria se refiere también a «la mejora de superficies forestales» y es ésta línea, que no tiene por qué implicar rapoblaciones arbóreas, la que encaja en áreas rurales como la que nos ocupa, donde no hay explotación agraria que reforestar. Por tanto, no se trata tampoco de desaprovechar las ayudas que para gestión de montes abre el Reglamento - otra discusión es la de su sentido en el marco de una política de compensación de rentas agrarias-, sino de no identificar siempre «mejora de superficies forestales» con reforestaciones.

La experiencia histórica acumulada en nuestro país en repoblaciones debe llevar al conjunto de instancias que intervienen en la gestión de los montes a una nueva concertación sobre el significado y las formas de intervenir en los espacios forestales actuales y en los que, por efecto del abandono de cultivos, puedan llegar a serlo. En ese sentido y si, como parece, la forestación de tierras agrarias definitivamente se orienta a espacios de vocación forestal o ya forestales, serían necesarios proyectos de carácter territorial en los que se definieran con claridad objetivos regionales y locales de fomento y regeneración de la cubierta vegetal, atendiendo al mantenimiento y desarrollo del patrimonio biológico, cultural y económico de los montes o de los predios que puedan llegar a serlo. En tales proyectos debieran incardinarse las iniciativas individuales, a diferencia de lo que viene ocurriendo, en general, con el programa de forestaciones, cuyo desarrollo está supeditado a actuaciones singulares de explotación, inconexas y sin integración en un modelo general del monte al que se aspira. Se trataría de fomentar y financiar operaciones no siempre vinculadas a la plantación de árboles, sino encaminadas también, según los casos, a la gestión del matorral mediterráneo.

En definitiva sería necesario integrar este tipo de actuaciones en una auténtica política forestal, algo que no ha existido hasta comienzos del pasado decenio, por más matices que puedan hacerse a esta afirmación. Así, desde la paralización de la estrategia forestal tradicional, basada en la repoblación generalizada de las cuencas vertientes de los embalses y de las áreas de elevada erosión potencial no ha habido una política para los montes en ninguna de las escalas territoriales y de decisión -europea, española y, lógicamente, regional-, pues no sólo se ha abandonado la forestación de nuevos espacios, sino que, prácticamente se han paralizado las necesarias intervenciones en las masivas re- 
poblaciones realizadas en las décadas anteriores.

En el caso de las más de más de 15.000 hectáreas que componen las repoblaciones del Alto Sorbe, algunas de la cuales tienen edades cercanas a los cincuenta años, únicamente se han realizado algunos tratamientos preventivos contra incendios forestales (cortafuegos y fajas protectoras alrededor de las vías de comunicación) lo cual ha generado un grave problema de excesiva espesura de las masas, ya que las plantaciones fueron realizadas con densidades casi siempre superiores a los $2.000 \mathrm{pies} / \mathrm{ha}$. La falta de tratamientos se ha convertido en una traba para la naturalización de las plantaciones pues actualmente son zonas prácticamente impenetrables en las que no se ha desarrollado el estrato arbustivo, pero también es un remora desde el punto de vista productivo ya que las repoblaciones «presentan un alto número de pies decrépitos y dominados y, en general, la esbeltez de los portes es muy acusada debido a la falta de luz, por la gran competencia» (J.C.M. 1998). Cabe destacarse también que la elevada biomasa de las repoblaciones incrementa de forma notable el riesgo de incendios forestales.

Frente a la inexistencia de política forestal, sí se ha implementado una importante política agropecuaria apoyada por abundantes fondos, lo que ha generado que espacios tradicionalmente forestales, sin otra vocación que la forestal, tengan que aprovechar los resquicios de la gigante política agraria para la gestión territorial de espacios desprovistos hoy de la función productiva de antaño. Las medidas de acompañamiento de la PAC se convierten entonces en una de las pocas vías de financiación con que cuentan los propietarios de espacios forestales que están dispuestos a dedicar su patrimonio inmueble a una función eminentemente ambiental.

No obstante, los últimos años marcan, a nuestro juicio, una inflexión en la política forestal, tanto en Europa como en numerosas comunidades autónomas españolas, varias de las cuales están elaborando y aprobando planes forestales de diverso signo. Los montes, por otra parte, están despertando de comienzos de los ochenta un interés académico y científico pluridisciplinar, donde van cobrado peso los aspectos socioecológicos, frente al sesgo dasocrático y productivista de épocas pasadas. Ese interés es un indicador más del protagonismo que recobra lo forestal en una etapa de crisis y agotamiento de políticas rurales de base sectorial y eminentemente agraria; en una etapa en la que toca repensar lo rural en términos más territoriales, ambientales y de equilibrio de usos 
y aprovechamientos, y en la que la reiterada multifuncionalidad de los espacios forestales adquiere un incuestionable valor estratégico desde una perspectiva rural más integradora y equilibrada que en el pasado. El Futuro del Mundo Rural (1988), aspectos ya citados de la reforma de la PAC de 1992 y, sobre todo, la Agenda 2000 - y explícitamente las referencias que en ella se hacen a las medidas forestales en un contexto rural amplio-son buenos exponentes de los cambios que a nivel comunitario se están produciendo en el proyecto de ruralidad europeo, y en el papel que en ese nuevo proyecto debe desempeñar lo forestal. En esa línea se mueve la muy reciente Comunicación de la Comisión al Consejo y al Parlamento (COM, 1998, 649-final) sobre «Una estrategia de la UE para el sector forestal», que - no lo olvidemos-es fruto de Resolución legislativa del Parlamento Europeo de 30 de enero de 1997, quien por primera vez en su historia, y con motivo de la cuestión forestal, se ha servido del derecho de iniciativa legislativa que le confiere el TUE.

El Dictamen del Comité Económico y Social de abril de 1997 sobre «Situación y problemas de la silvicultura en la Unión Europea y potencial de desarrollo de las políticas forestales» —un documento de mucho interés a nuestro modo de ver-se ubica con realismo en el terreno de los valores «sociales, económicos y ecológicos de los bosques», y en pos de una estrategia que los potencie conjuntamente, allí donde sea posible, todo ello presidido por la tesis de que «la explotación de todos los recursos del sector forestal es vital para la preservación de la vida rural activa».

El relativo impulso que parece haber tomado de nuevo la política forestal se ha concretado en el Alto Sorbe en la redacción de un «Plan de intervenciones selvícolas en las repoblaciones» que sin integrar en su totalidad las últimas propuestas sobre estrategia forestal, propone la realización de claras sobre los montes repoblados de la zona con el fin de mejorar sus condiciones de crecimiento e, indirectamente, facilitar la naturalización de las masas.

\section{BIBLIOGRAFÍA}

ABELLO, M. A. (1985); Historia y evolución de las repoblaciones forestales en España. Universidad Complutense, 749 pág.

CASTEL, C. (1873); «Noticias físico-naturales de la sección N.O. de la provincia de Guadalajara», Revista forestal, económica y agrícola. Tomo VII, 1873-1874. Madrid, pp. 216-225, 254-258. 
- (1881); Descripción física, geognóstica, agrícola y forestal de la provincia de Guadalajara. Guadalajara. Reproducción facsimil, (1998). Edición realizada por Antonio Morcillo.

Fidalgo HiJano, C. (1987); La transformación humana del paisaje en la Serranía de Atienza. Madrid. Ediciones de la Universidad Autónoma de Madrid.

GARCíA RUIZ, J. M. (1988); «La repoblación forestal en la provincia de Logroño», Cuadernos de Investigación (Geografía e Historia), n. ${ }^{\circ} 2$, pp. 25-36.

Gómez Mendoza, J, Mata Olmo, R. (1992); «Actuaciones forestales públicas desde 1940. Objetivos, criterios y resultados»; Agricultura y Sociedad, n. ${ }^{\circ}$ 65, pp. 15-64.

LÓPEZ GÓMEz, A. (1974): «Colectivismo y sistemas agrarios en la serranía de Atienza (Guadalajara)». Estudios Geográficos, n. ${ }^{\circ}$ 137, pp. 519-578.

Manuel, C. M. (1996); Tierras y montes públicos en la sierra de Madrid. Madrid, M.A.P.A.

ORTIGOSA, L. (1991); Las repoblaciones forestales en La Rioja: resultados y efectos geomorfológicos. Logroño, Geoforma ediciones.

ORTUÑo, F. (1990); «El plan para la repoblación forestal de España del año 1939. Análisis y comentarios», Ecología, Fuera de serie, pp. 373-392.

REDONDo, M. (1989); «La repoblación forestal en los montes de Toledo», Congreso Nacional de Geografía, vol. 2, pp. 228-237.

Rico, E. (1995); Política forestal e repobacions en Galicia 1941-1971. Tesis doctoral inédita.

SÁEz Poмвo (1998): Propiedad pública, organización del territorio y evolución del paisaje en la Sierra Norte de Madrid. Tesis doctoral inédita. Universidad Autónoma de Madrid.

\section{FUENTES}

A.M.T. (1891); «Título de propiedad del Monte de Abajo y terrenos llamados Tras del Lomo, Cerrajo y Piedra Blanca». Archivo Municipal de Tamajón.

A.M.T. (1899); «Contrato de cesión de pastos y bases para la administración y cobranza de los mismos de 1899. Término Municipal de Tamajón». Archivo Municipal de Tamajón.

A.M.T. (1907); «Expediente de repartimiento de pastos de la Villa de Tamajón». Archivo Municipal de Tamajón.

A.M.T. (1935); «Expediente de repartimiento de pastos de la Villa de Tamajón». Archivo Municipal de Tamajón.

A.M.T. (1964); «Escrito de la Hermandad de Labradores y Ganaderos de Tamajón, en representación de los propietarios de las tierras de Baldíos, al Sr. Director General del Patrimonio Forestal del Estado». Archivo Municipal de Tamajón.

A.M.T. (1976); «Expediente de Expropiación Forzosa del predio denominado Palancares» I.C.O.N.A. Archivo Municipal de Tamajón.

A.M.V. (1903); «Actos de conciliación con conveniencia de los terrenos Ocejón, Mojón Blanco y Umbría Gorda, Peyuste, Rincón y Dehesa del Sonsaz».

A.M.V. (1924); «Copia de la escritura de Mojón Blanco y Canaleja. Valverde de los Arroyos». 1924.

A.H.G. (1887); «Expediente de Tasación de una Dehesa llamada Cespedones. Propios. Rústicas, Menor Cuantía, Palancares». Archivo Histórico Provincial de Guadalajara.

ICONA (1975); «Expediente de la compra del monte de Palancares», Archivo Dirección General de Conservación de la Naturaleza.

J.C.C.M. (1998); «Plan de intervenciones selvícolas en las repoblaciones de la unidad natural Sierra de Ayllón, Guadalajara». Delegación de Agricultura y Medio Ambiente, TRAGSATEC. 
LIBRO... (1939); «Libro para apuntes de los cobros y ajustes de la Dehesa de Cespedones y Monte de este pueblo». 1939-1967» Documento conservado por un vecino.

P.F.E. (1951); «Expediente del Consorcio del monte Robledo y Otros Jócar», Archivo Dirección General de Conservación de la Naturaleza.

P.F.E. (1951a); «Expediente del Consorcio del monte Tejera Negra y Renovizal», Archivo Dirección General de Conservación de la Naturaleza.

P.F.E. (1952); «Expediente del Consorcio del monte Bienes Comunales de Semillas», Archivo Dirección General de Conservación de la Naturaleza.

P.F.E. (1955); «Proyecto de repoblación de diversos montes con necesidad de repoblación obligatoria en el término de Cantalojas», Patrimonio Forestal del Estado, Brigada Ávila, Segovia, Guadalajara. Archivo Dirección General de Conservación de la Naturaleza.

P.F.E. (1943); «Memoria general de reconocimiento de la cuenca del río Sorbe», $4 .^{a} \mathrm{Di}-$ visión Hidrológico Forestal. Archivo Dirección General de Conservación de la Naturaleza.

P.F.E. (1963); «Proyecto de repoblación forestal del monte Robledo y otros, término municipal de Arbancón; anejo Jócar». Archivo Distrito Forestal de Guadalajara.

P.F.E. (1967); «Proyecto de repoblación de diversos montes con necesidad de repoblación obligatoria en la cuenca alimentadora del embalse de Beleña. Término municipal de Arbancón». Archivo Dirección General de Conservación de la Naturaleza.

P.F.E. (1968); «Memoria de reconocimiento general de la cuenca de la presa de Beleña de Sorbe, sobre el río Sorbe», Servicio Hidrológico-Forestal de Guadalajara, $1^{a}$ Inspección Hidrológico-Forestal. Archivo Dirección General de Conservación de la Naturaleza.

\section{ABREVIATURAS}

A.H.G. Archivo histórico provincial de Guadalajara.

A.M.T. Archivo Municipal de Tamajón.

A.M.V. Archivo Municipal de Valverde de los Arroyos.

J.C.C.M. Junta de Comunidades de Castilla La Mancha.

P.F.E. Patrimonio Forestal del Estado.

RESUMEN: El artículo da cuenta de papel desempeñado por las sociedades de vecinos en las opciones en las operaciones repobladoras desarrolladas en la cuenca alta del río Sorbe, al norte de la provincia de Guadalajara, durante el último medio siglo. Para ello se comparan las actuacciones masivas de los años 50 a 70, con la aplicación de las recientes medidas de reforestación de tierras agrícolas, de acompañamiento de la reforma de la P.A.C. aprobada en 1992. El estudio de caso realizado es también una buena oportunidad para reflexionar sobre el cambiante significado de los montes de vecinos a lo largo de una etapa de profunda transformación socioeconómica y política.

SUMMARY: The article sumarizes the role of communal land associations in reafforestation operations held during the last fifty years in the river Sorbe basin, located north of the Guadalajara province. For this purpose, masive actuations wich took place during the years 1950 to 1970 are compared to recent agricole reafforestation policies wich were included in the P.A.C. reform approved in 1992. This case-study provides the oportunity to discuss the variable significance of communal forest during a period of deep social, economic and political transformations.

PALABRAS CLAVE: Repoblaciones, política forestal, montes, bosques, montes comunales, política agraria comunitaria, Ayllón. 